\title{
PET-Plan: potential for dose escalation by target volume reduction in locally advanced NSCLC
}

\author{
Evelyn E. C. de Jong ${ }^{1}$, Janita E. van Timmeren ${ }^{2}$ \\ ${ }^{1}$ Department of Radiotherapy, Catharina Hospital, Eindhoven, The Netherlands; ${ }^{2}$ Department of Radiation Oncology, University Hospital Zürich \\ and University of Zürich, Zürich, Switzerland \\ Correspondence to: Evelyn E. C. de Jong, PhD. Department of Radiotherapy, Catharina Hospital, Michelangelolaan 2, 5623 EJ, Eindhoven, The \\ Netherlands. Email: evelyn.d.jong@catharinaziekenhuis.nl. \\ Provenance and Peer Review: This article was commissioned by the editorial office, Translational Lung Cancer Research. The article did not undergo \\ external peer review. \\ Comment on: Nestle U, Schimek-Jasch T, Kremp S, et al. Imaging-based target volume reduction in chemoradiotherapy for locally advanced non- \\ small-cell lung cancer (PET-Plan): a multicentre, open-label, randomised, controlled trial. Lancet Oncol 2020;21:581-92.
}

Submitted May 14, 2020. Accepted for publication May 27, 2020.

doi: $10.21037 /$ tlcr-20-653

View this article at: http://dx.doi.org/10.21037/tlcr-20-653

The standard treatment for (inoperable) locally advanced (LA) non-small cell lung cancer (NSCLC) is concurrent chemoradiotherapy (CCRT), showing improved survival over sequential chemotherapy and radiotherapy (RT) or RT alone (1). The development of intensity-modulated RT (IMRT) resulted in more conformal treatment plans than three-dimensional conformal RT (3DCRT), thus allowing for increased dose to the target while avoiding surrounding normal tissue. In addition, improvements in treatment planning systems and image-guided RT (IGRT) have contributed to more effective RT. Nevertheless, besides death from the development of distant metastases, local progression is the main issue currently resulting in poor outcomes for LA-NSCLC (2). Therefore, the effectiveness of RT is largely dependent on the accurate assessment of the localization and extend of the target volume. Thus, higher effectiveness of RT to improve local tumor control could be achieved by both reduction of the target volume and dose escalation. In May 2009, a multicenter, randomized controlled trial (RCT) was started to investigate the restriction of RT to ${ }^{18} \mathrm{~F}$-fluorodeoxyglucose positron emission tomography $\left({ }^{18} \mathrm{~F}-\mathrm{FDG}-\mathrm{PET}\right)$ positive areas. The results of the RCT were recently presented in an article published in Lancet Oncology (March 12, 2020), entitled "Imaging-based target volume reduction in chemoradiotherapy for locally advanced non-small-cell lung cancer (PET-Plan): a multicentre, open-label, randomised, controlled trial" (3).
In this RCT called PET-Plan, a total of 205 patients were eligible for randomization between the PET-based target group, i.e., informed by ${ }^{18} \mathrm{~F}$-FDG-PET alone, or the conventional target group, i.e., informed by ${ }^{18} \mathrm{~F}-\mathrm{FDG}-\mathrm{PET}$ and computed tomography (CT) in which elective nodal irradiation was applied as well, to test for non-inferiority of PET-based planning. The primary endpoint of the study was locoregional regression. Secondary endpoints included out-of-field progression, time to in-field progression, time to distant progression, overall survival (OS), progressionfree survival, acute treatment-related toxicity and late treatment-related toxicity. The rationale for this study was to generate prospective evidence for target volume reduction based on ${ }^{18} \mathrm{~F}$-FDG-PET, offering potential to safe dose escalation, which might lead to improved local control without increased toxicity.

Historically, elective nodal irradiation has been performed to eradicate lymph nodes that might contain micrometastases. This approach originates from before non-invasive imaging has been used for treatment planning and the benefits of elective nodal irradiation have never been proven. Locoregional recurrences are predominantly observed within the primary tumor and less frequently in the involved lymph nodes (4). Moreover, the dose distribution outside the planning target volume (PTV) might 'accidently' irradiate lymph nodes, therefore reducing the risk of recurrence (5). Since elective nodal irradiation 
additionally limits dose due to the risk of developing adverse events (e.g., radiopneumonitis), it has been proposed to favor in-field RT over elective nodal irradiation, although largely dependent, amongst others, on the accuracy of staging (6). Even though ${ }^{18} \mathrm{~F}$-FDG-PET was originally established for staging, integration of ${ }^{18} \mathrm{~F}-\mathrm{FDG}-\mathrm{PET}$ into treatment planning for radiation target definition has been proposed and investigated in multiple studies because of its high diagnostic accuracy in selecting involved lymph nodes (7). However, by using only ${ }^{18} \mathrm{~F}-\mathrm{FDG}-\mathrm{PET}$ to determine the radiation target volume, PET-negative lymph nodes that are enlarged on CT are no longer irradiated. The most recent European Organization for Research and Treatment of Cancer (EORTC) guidelines recommends selective nodal irradiation on the basis of $\mathrm{CT}$ and ${ }^{18} \mathrm{~F}$-FDG-PET, but including information from endobronchial or oesophageal ultrasound with needle aspiration and mediastinoscopy, if available, to achieve the highest sensitivity and specificity (7). In PET-Plan, both ${ }^{18}$ F-FDG-PET positive lymph nodes and pathologically confirmed lymph nodes were included in the nodal clinical target volume (CTV). For the conventional target group in PET-Plan, the atelectasis and CT positive nodes were added to the PET-based gross tumor volume (GTV). The trial shows a potential benefit of reducing radiation target volume based on ${ }^{18} \mathrm{~F}$-FDG-PET: a hazard ratio of 0.57 for locoregional control was observed in the per-protocol set. The cumulative incidence for locoregional progression at 2 years was $20 \%$ in the PET-based target group compared to $39 \%$ for the conventional target group. The out-of-field progression, in-field progression, distance progression, overall survival and progression-free survival were not significantly different between the PET-based target group and the conventional target group.

When using ${ }^{18}$ F-FDG-PET for treatment planning, standardized protocols are required to achieve high diagnostic accuracy. To this extend, quality assurance is highly recommended, which was extensively performed in the PET-Plan study for both planning and imaging (3). To achieve consistent and comparable ${ }^{18} \mathrm{~F}-\mathrm{FDG}-\mathrm{PET}$ imaging, timing is very important. The time interval between the ${ }^{18} \mathrm{~F}-\mathrm{FDG}-\mathrm{PET}$ scan acquisition and the start of RT should be as short as possible ( $<4$ weeks) to avoid false negative results (8). Regional recurrences could be an effect of understaging due to a too long time interval between the staging ${ }^{18} \mathrm{~F}$-FDG-PET scan and the start of the RT (8). The other aspect of ${ }^{18}$ F-FDG-PET scanning in which timing is important is the time between ${ }^{18} \mathrm{~F}$-FDG injection and the start of the PET scan acquisition. The standardized uptake value (SUV) keeps increasing until 60 minutes after injection, but at a certain moment in time thereafter, decreases again. When using autosegmentation based on the SUV, it is important to have a standardized time between ${ }^{18} \mathrm{~F}$-FDG injection and start scanning. Besides timing, the threshold to determine whether a lymph node is considered positive or negative influences the sensitivity and specificity of ${ }^{18} \mathrm{~F}$-FDG-PET (9). Other parameters that influence the diagnostic accuracy of ${ }^{18} \mathrm{~F}$-FDG-PET are the scanner, injected ${ }^{18} \mathrm{~F}-\mathrm{FDG}$ dose, and the applied image corrections (9).

However, when used appropriately, ${ }^{18} \mathrm{~F}$-FDG-PET can define target volumes for RT with high diagnostic accuracy, allowing for reduced target volumes as shown in PET-Plan. This, subsequently, enables dose escalation without increasing the risk of side effects. Locoregional recurrence was shown to be primarily located in the high FDG uptake region of the primary tumor of the pretreatment ${ }^{18} \mathrm{~F}$-FDG-PET scan, also suggesting for dose escalation on the primary tumor (10). Current clinical practice for LA-NSCLC is a CCRT, with an RT dose of 60 Gy given in 2 Gy once daily fractions over 6 weeks $(11,12)$, but several studies investigated safety and outcome of dose escalation. In the pilot study of the PET-Plan trial, the total dose was escalated above 66.6 Gy in steps of 1.8 Gy until 73.8 Gy (13). Low toxicity rates and a median OS of 19.2 months were observed, and dose escalation was shown to be safe with regard to out-of-field progression (13). The PET-Plan study showed that the mean escalated RT dose was significantly higher in the PET-based target group (67.3 Gy), than in the conventional target group (65.3 Gy) (3). Next to that, $47 \%$ of patients in the PETbased target group received more than 68 Gy, versus 33\% in the conventional target group. In summary, in PETPlan, dose escalation showed no negative effects on survival or toxicity. Nevertheless, no relation between locoregional regression and dose was observed (3).

The PET-Plan trial was performed around the same time and in a similar population as the RTOG 0617 trial (11). In the RTOG 0617 trial the median OS of patients in the conventional dose group, receiving $30 \times 2$ Gy, was significantly longer (28.7 months) than that of the patients in the high-dose group, receiving $37 \times 2$ Gy (22.9 months). In the RTOG 0617 trial patients were randomized between two dose levels, whereas PET-Plan applied individual isotoxic dose escalation. In the RTOG 0617 trial mean 
lung dose (MLD), esophageal dose and heart dose were all significantly higher in the high-dose group. Increased heart dose is the most likely explanation for the worse outcome in patients given $74 \mathrm{~Gy}$. Another reason for the negative outcome of the RTOG 0617 trial could be the prolonged overall treatment time. It is shown that a treatment duration beyond six weeks ( 30 fractions), results in a $1.6 \%$ survival loss for each additional treatment day, probably caused by the rapid repopulation of NSCLCs (14). Therefore, hypofractionation seems a promising approach for dose escalation. A recent phase II randomized trial showed a median OS of 31.5 months for patients receiving 24 fractions of $2.75 \mathrm{~Gy}$ (15). Hypofractionation was also investigated in the phase II randomized PET-boost trial, in which patients were randomized that were eligible for receiving 24 fractions of $>3$ Gy. In this trial, isotoxic dose escalation of the high FDG-uptake region ( $>50 \%$ of SUVmax) inside the primary tumor was compared to dose escalation of the entire primary tumor, forcing equal MLD in both arms $(4,10)$. The mean dose inside the boost volume of the primary tumor boost group was 79.2 Gy while in the PET boost group 86.9 Gy. No significant dose difference for the organs at risk (OARs) was seen between both groups. Dose limiting organs were the mediastinal structures and the brachial plexus. However, higher acute and late toxicity in the PET boost group was seen compared to the primary tumor boost group.

Dose escalation is limited by normal tissue dose constraints, so it is important to compare toxicity with outcome for different fractionation schemes. The variety in OAR constraints could explain the differences in dose escalation levels reached in the different trials.

To conclude, this multicenter RCT PET-Plan is the first study providing evidence for improving the effectiveness of RT by target volume reduction using ${ }^{18} \mathrm{~F}$-FDG-PET in treatment planning, even though superiority has not yet been confirmed. This result will probably affect current clinical practice, and according to the study, PET-based target definition could be implemented as standardof-care. Upon full integration, standardized ${ }^{18} \mathrm{~F}-\mathrm{FDG}$ PET protocols to treatment planning are essential, and quality assurance is warranted. When used appropriately, ${ }^{18}$ F-FDG-PET can define target volumes for RT with high diagnostic accuracy, offering potential for dose escalation without increased risk of toxicity. The results on the effect of dose escalation in PET-Plan, however, are not likely to change clinical practice yet. Discrepancies between recent randomized trials regarding dose escalation show that the best approach for dose escalation is yet still to be unraveled.

\section{Acknowledgments}

Funding: None.

\section{Footnote}

Conflicts of Interest: Both authors have completed the ICMJE uniform disclosure form (available at http://dx.doi. org/10.21037/tlcr-20-653). The authors have no conflicts of interest to declare.

Ethical Statement: The authors are accountable for all aspects of the work in ensuring that questions related to the accuracy or integrity of any part of the work are appropriately investigated and resolved.

Open Access Statement: This is an Open Access article distributed in accordance with the Creative Commons Attribution-NonCommercial-NoDerivs 4.0 International License (CC BY-NC-ND 4.0), which permits the noncommercial replication and distribution of the article with the strict proviso that no changes or edits are made and the original work is properly cited (including links to both the formal publication through the relevant DOI and the license). See: https://creativecommons.org/licenses/by-nc-nd/4.0/.

\section{References}

1. Curran WJ Jr, Paulus R, Langer CJ, et al. Sequential vs. concurrent chemoradiation for stage III non-small cell lung cancer: randomized phase III trial RTOG 9410. J Natl Cancer Inst 2011;103:1452-60.

2. Machtay M, Paulus R, Moughan J, et al. Defining local-regional control and its importance in locally advanced non-small cell lung carcinoma. J Thorac Oncol 2012;7:716-22.

3. Nestle U, Schimek-Jasch T, Kremp S, et al. Imagingbased target volume reduction in chemoradiotherapy for locally advanced non-small-cell lung cancer (PET-Plan): a multicentre, open-label, randomised, controlled trial. Lancet Oncol 2020;21:581-92.

4. van Diessen J, De Ruysscher D, Sonke JJ, et al. The acute and late toxicity results of a randomized phase II doseescalation trial in non-small cell lung cancer (PET-boost trial). Radiother Oncol 2019;131:166-73. 
5. De Ruysscher D, Wanders S, van Haren E, et al. Selective mediastinal node irradiation based on FDG-PET scan data in patients with non-small-cell lung cancer: a prospective clinical study. Int J Radiat Oncol Biol Phys 2005;62:988-94.

6. Belderbos JS, Kepka L, Spring Kong FM, et al. Report from the International Atomic Energy Agency (IAEA) consultants' meeting on elective nodal irradiation in lung cancer: non-small-Cell lung cancer (NSCLC). Int J Radiat Oncol Biol Phys 2008;72:335-42.

7. De Ruysscher D, Faivre-Finn C, Moeller D, et al. European Organization for Research and Treatment of Cancer (EORTC) recommendations for planning and delivery of high-dose, high precision radiotherapy for lung cancer. Radiother Oncol 2017;124:1-10.

8. Vanneste BG, Haas RL, Bard MP, et al. Involved field radiotherapy for locally advanced non-small cell lung cancer: isolated mediastinal nodal relapse. Lung Cancer 2010;70:218-20.

9. Schmidt-Hansen M, Baldwin DR, Hasler E, et al. PETCT for assessing mediastinal lymph node involvement in patients with suspected resectable non-small cell lung cancer. Cochrane Database Syst Rev 2014;2014:Cd009519.

10. van Elmpt W, De Ruysscher D, van der Salm A, et al. The PET-boost randomised phase II dose-escalation trial in non-small cell lung cancer. Radiother Oncol

Cite this article as: de Jong EEC, van Timmeren JE. PETPlan: potential for dose escalation by target volume reduction in locally advanced NSCLC. Transl Lung Cancer Res 2020;9(4):15951598. doi: $10.21037 /$ tlcr-20-653
2012;104:67-71.

11. Bradley JD, Paulus R, Komaki R, et al. Standard-dose versus high-dose conformal radiotherapy with concurrent and consolidation carboplatin plus paclitaxel with or without cetuximab for patients with stage IIIA or IIIB non-small-cell lung cancer (RTOG 0617): a randomised, two-by-two factorial phase 3 study. Lancet Oncol 2015;16:187-99.

12. Postmus PE, Kerr KM, Oudkerk M, et al. Early and locally advanced non-small-cell lung cancer (NSCLC): ESMO Clinical Practice Guidelines for diagnosis, treatment and follow-up. Ann Oncol 2017;28:iv1-iv21.

13. Fleckenstein J, Hellwig D, Kremp S, et al. F-18-FDG-PET confined radiotherapy of locally advanced NSCLC with concomitant chemotherapy: results of the PET-PLAN pilot trial. Int J Radiat Oncol Biol Phys 2011;81:e283-9.

14. Mehta M, Scrimger R, Mackie R, et al. A new approach to dose escalation in non-small-cell lung cancer. Int J Radiat Oncol Biol Phys 2001;49:23-33.

15. Walraven I, van den Heuvel M, van Diessen J, et al. Longterm follow-up of patients with locally advanced non-small cell lung cancer receiving concurrent hypofractionated chemoradiotherapy with or without cetuximab. Radiother Oncol 2016;118:442-6. 